\title{
Adaptive Neural Network Control of Serial Variable Stiffness Actuators
}

\author{
Zhao Guo, ${ }^{1}$ Yongping Pan, ${ }^{2,3}$ Tairen Sun, ${ }^{4}$ Yubing Zhang, ${ }^{1}$ and Xiaohui Xiao ${ }^{1}$ \\ ${ }^{1}$ School of Power and Mechanical Engineering, Wuhan University, Wuhan 430072, China \\ ${ }^{2}$ Department of Biomedical Engineering, National University of Singapore, Singapore \\ ${ }^{3}$ National University of Singapore (Suzhou) Research Institute, Suzhou 215123, China \\ ${ }^{4}$ Department of Electrical and Information Engineering, Jiangsu University, Zhenjiang 212013, China \\ Correspondence should be addressed to Yongping Pan; yongppan@gmail.com
}

Received 13 August 2017; Revised 8 September 2017; Accepted 20 September 2017; Published 8 November 2017

Academic Editor: Junpei Zhong

Copyright ( 2017 Zhao Guo et al. This is an open access article distributed under the Creative Commons Attribution License, which permits unrestricted use, distribution, and reproduction in any medium, provided the original work is properly cited.

\begin{abstract}
This paper focuses on modeling and control of a class of serial variable stiffness actuators (SVSAs) based on level mechanisms for robotic applications. A multi-input multi-output complex nonlinear dynamic model is derived to fully describe SVSAs and the relative degree of the model is determined accordingly. Due to nonlinearity, high coupling, and parametric uncertainty of SVSAs, a neural network-based adaptive control strategy based on feedback linearization is proposed to handle system uncertainties. The feasibility of the proposed approach for position and stiffness tracking of SVSAs is verified by simulation results.
\end{abstract}

\section{Introduction}

Variable stiffness actuators (VSAs), which can increase safety in physical human-robot interaction and meet the dynamic requirements of robots in unknown environments, have been developed for a new generation of robots [1-11]. VSAs present advantages in terms of mechanical stiffness adjusting, energy storing, and force sensing capabilities [3]. According to the design configurations, there are two typical mechanical arrangements for VSAs: one is the antagonistic configuration with a pair of actuators coupled via nonlinear springs [10], and the other is the serial configuration with two independent motors, where a principal motor drives the joint position through a compliant transmission, and a secondary motor adjusts the stiffness [11].

Antagonistic-type VSAs need two driving units to change the stiffness and position synchronously, which lead to high energy consumption and complex control design. Serialtype VSAs (SVSAs) do not have this requirement and have gained more attention. Recently, new types of SVSAs have been developed based on level arm mechanisms, where the mechanical stiffness is regulated by moving one of the level points: a pivot point, a spring located point, or a force point [12-14]. Thus, the SVSAs are not significantly influenced by the coupling between the load and the stiffness transmission mechanism. It also brings higher energy efficiency in unloaded conditions [15]. Based on the variable ratio lever principle by changing the pivot position, a novel compact rotational SVSA with an Archimedean spiral relocation mechanism (ASRM) was developed to obtain compact mechanical structure, large adjustable stiffness range, and better force transmission ability in [15]. In this innovative design, the ASRM is applied to transfer the rotation of the Archimedean spiral cam into the linear motion of the pivot, and an elastic force transmission mechanism with a spring shaft vertical to the output link is proposed to achieve large deflection angle and high energy storage ability.

VSAs are multi-input multioutput (MIMO), highly coupled, and complex nonlinear systems which include both structured and unstructured uncertainties [16]. In VSAs, the stiffness variation brings physical modifications, requiring the control system to quickly transit among different operating conditions. In addition, the coupling between stiffness and positioning mechanisms and the increased system dimensionality complicate the entire control system [16]. As a result, it is challenging to design control strategies for VSAs 
in robotic applications. Different control strategies have been proposed to for VSAs, where the simplest one is the PD control in [16]. However, the performance of the PD control highly depends on $\mathrm{PD}$ gains due to the high nonlinearity of VSAs. In [17, 18], a classical nonlinear control technique termed feedback linearization was explored for VSAs. Nevertheless, feedback linearization is very sensitive to modeling accuracy so that significant efforts in system modeling and parameter identification are required to achieve a desired performance. Based on the feedback linearization, some advanced control methods, such as gain-scheduling control [19], active damping control [20], backstepping control [21], nonlinear model predictive control [22], and output feedback control [23], have been integrated to improve stiffness and position tracking performances of VSAs. Although these control methods are proved to be effective for VSAs, they may exhibit undesired performances especially if the VSA dynamics are highly nonlinear and experience large parameter variations due to the changes of the actuator stiffness and load conditions [24].

Neural networks (NNs) have been widely applied to control complex nonlinear systems, especially complex robotic systems in recent years [25-37]. Compared with the traditional adaptive control, NN adaptive control (NNAC) has two prominent attractive features [38]. Firstly, due to the universal approximation property of NNs, the difficulty of system modeling in many practical control problems can be largely alleviated. Secondly, due to the practical persistently exciting (PE) condition of NNs, parameter convergence is easier to be obtained during control resulting in an exact online modeling and superior exponential tracking. Although NNAC is promising for complex nonlinear systems like VSAs, it is seldom applied to VSAs except [39], where antagonistic VSAs are considered in [39].

This paper focuses on modeling and control of a class of SVSAs based on level mechanisms. Firstly, a MIMO complex nonlinear dynamic model is derived to fully describe SVSAs and the relative degree of the model is determined accordingly; secondly, based on the feedback linearization, a direct NNAC strategy is proposed to govern the SVSA model; finally, high-fidelity simulations are provided to verify the effectiveness of the proposed approach. Compared with other control strategies for VSAs, the proposed NNAC can deal with high coupling and parametric uncertainties of SVSAs. In addition, the control on both position and stiffness tracking remain effective under load changing conditions.

\section{Serial Variable Stiffness Actuator Modeling}

As illustrated in Figure 1, a compact rotational SVSA consists of a variable stiffness mechanism (VSM), a principal motor, and a secondary motor, where the principal motor drives the output link motion through the spring transmission, and a secondary motor adjusts the stiffness of the actuator by changing the position of the pivot with an ASRM [15].

By considering gravity and external loads, the dynamic model of SVSAs is represented as follows:

$$
\begin{gathered}
M \ddot{q}+D \dot{q}+\tau_{e}\left(\theta_{2}, \varphi\right)+\tau_{g}(q)=\tau_{\text {ext }} \\
B_{1} \ddot{\theta}_{1}+D_{1} \dot{\theta}_{1}-\tau_{e}\left(\theta_{2}, \varphi\right)=u_{1} \\
B_{2} \ddot{\theta}_{2}+D_{2} \dot{\theta}_{2}+\tau_{r}\left(\theta_{2}, \varphi\right)=u_{2},
\end{gathered}
$$

where $q$ is a position of the output link, $\theta_{i}$ with $i=1,2$ is a position associated with each motor, $\varphi:=q-\theta_{1}$ is a deflection angle of the elastic transmission, $M$ is an inertia of the output link, $B_{i}$ is a reflected inertia of each motor, $D$ is a reflected damping of the link, $D_{i}$ is a reflected damping of each motor, $\tau_{g}(q)$ is a gravity torque, $u_{i}$ is a control input of each motor, $\tau_{\text {ext }}$ is an external torque, $\tau_{r}$ is a coupling reaction torque, and $\tau_{e}$ is an elastic torque of the spring transmission.

The elastic torque across the transmission is given by

$$
\tau_{e}=K_{s} R^{2} \mu^{2} \frac{\sin \varphi \cos \varphi}{(1-\mu \cos \varphi)^{2}}
$$

where $K_{s}$ is a spring stiffness, $R$ is a radius of the output link, and $\mu$ is a lever length ratio. The coupled flexibility torque, representing the transmission deformation reacting on the secondary motor, is given by

$$
\tau_{r}=K_{s} R^{2} a^{2} \frac{\sin 2 \beta \sin ^{2} \varphi}{2(R-a \cos \varphi)\left(a^{2}+R^{2}-2 a R \cos \varphi\right)},
$$

where $\beta=\arctan \left(-\theta_{2} / \gamma\right)$ is a tangent angle of the Archimedean spiral gear, $\gamma$ is a reduction gear ratio of the secondary motor, and $a=\mu R=R \theta_{2} / 2 \pi$ is a distance from the pivot point to the joint center. The stiffness of this SVSA is formulated by

$$
\sigma\left(\theta_{2}, \varphi\right)=\frac{\partial \tau_{e}\left(\theta_{2}, \varphi\right)}{\partial \varphi}=K_{s} R^{2} \mu^{2} \frac{\cos 2 \varphi-\mu \cos \varphi}{(1-\mu \cos \varphi)^{3}}
$$

The level length ratio $\mu$ can be represented by the position of the secondary motor as follows:

$$
\mu=\frac{\theta_{2}}{2 \pi \gamma}+\mu_{0}
$$

where $\mu_{0}$ is an initial level length ratio.

\section{Neural Network Control Design}

3.1. Problem Formulation. The dynamic model of SVSAs given by (1)-(5) can be represented in the standard form

$$
\begin{aligned}
& \dot{\mathbf{x}}=\mathbf{f}(\mathbf{x})+\mathbf{g}(\mathbf{x}) \mathbf{u} \\
& \mathbf{y}=\mathbf{h}(\mathbf{x}),
\end{aligned}
$$




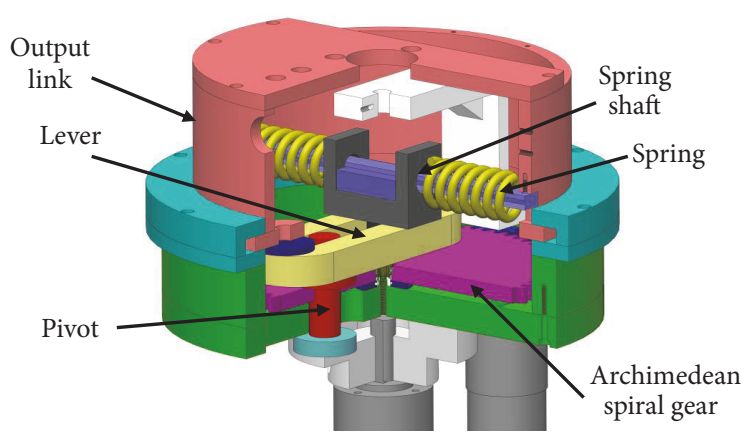

(a)

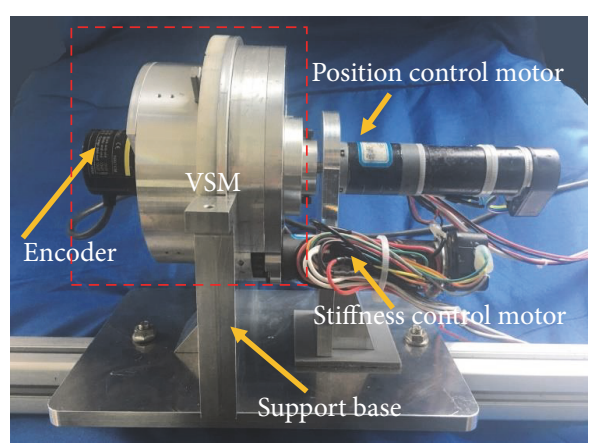

(b)

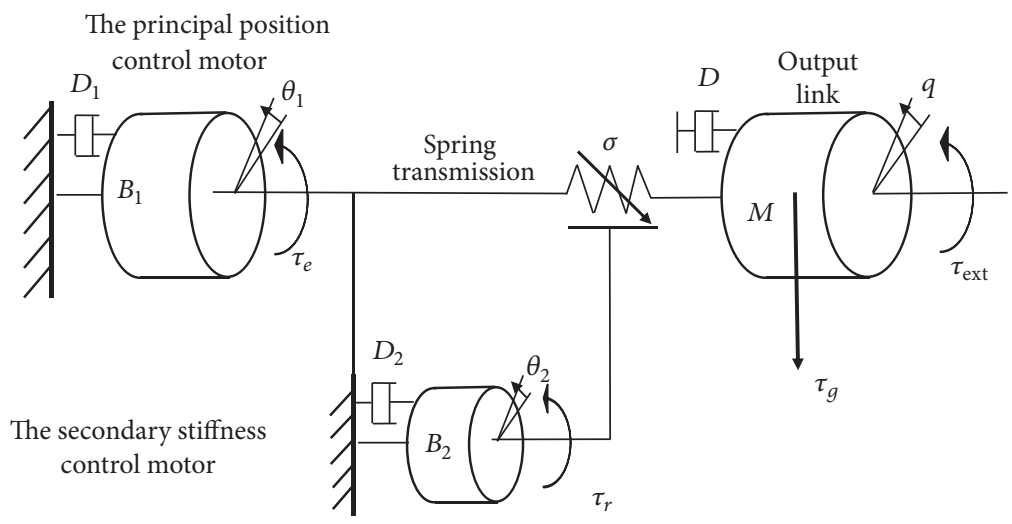

(c)

FIGURE 1: CAD model (a), prototype (b), and schematic model (c) of the SVSA.

where $\mathbf{x}=\left[q, \dot{q}, \theta_{1}, \dot{\theta}_{1}, \theta_{2}, \dot{\theta}_{2}\right]^{T}$ is a vector of system states, $\mathbf{u}=\left[u_{1}, u_{2}\right]^{T}$ is a control input, $y=[q, \sigma]^{T}$ is a system output, and

$$
\begin{aligned}
& \mathbf{f}(\mathbf{x})=\left[\begin{array}{c}
\dot{q} \\
M^{-1}\left(-D \dot{q}-\tau_{e}+\tau_{\mathrm{ext}}-\tau_{g}\right) \\
\dot{\theta}_{1} \\
B_{1}^{-1}\left(-D_{1} \dot{\theta}_{1}+\tau_{e}\right) \\
\dot{\theta}_{2} \\
\\
B_{2}^{-1}\left(-D_{2} \dot{\theta}_{2}-\tau_{r}\right)
\end{array}\right], \\
& \mathbf{g}(\mathbf{x})=\left[\begin{array}{cc}
0 & 0 \\
0 & 0 \\
0 & 0 \\
B_{1}^{-1} & 0 \\
0 & 0 \\
0 & B_{2}^{-1}
\end{array}\right], \\
& \mathbf{h}(\mathbf{x})=\left[\begin{array}{c}
q \\
\sigma
\end{array}\right] .
\end{aligned}
$$

The equilibrium point of system (6) is $\mathbf{x}_{0}=0$. Let $q_{d} \in$ $R$ and $\sigma_{d} \in R$ be bounded desired outputs. Define tracking errors $e_{q}:=q-q_{d}$ and $e_{\sigma}:=\sigma-\sigma_{d}$.

Assumption 1. There exist uncertainties in $\mathbf{f}(\mathbf{x})$ and $\mathbf{g}(\mathbf{x})$ due to modeling difficulties and modeling errors.

Assumption 2. $\ddot{q}_{d}, \dddot{q}_{d}, \dddot{q}_{d}, \dot{\sigma}_{d}, \ddot{\sigma}_{d} \in R$ exist and are bounded.

The objective of this study is to design NNAC law without the exact knowledge of the SVSA model (1) such that the tracking errors $e_{q}$ and $e_{\sigma}$ are converging.

\subsection{Feedback Linearization-Based Control}

Definition 3 (the standard Lie derivative notation is used for the description [40]). The relative degree from the input $\mathbf{u}$ to the output $\mathbf{y}$ of system (6) at the equilibrium $\mathbf{x}_{0}$ is $\left(\sigma_{1}, \sigma_{2}\right)$ if (a) $L_{g_{j}} L_{f}^{k} h_{i}(x)=0(1 \leq j, i \leq 2)$ for all $k<\sigma_{i}-1$ and all $\mathbf{x}$ in a neighborhood of $\mathbf{x}_{0}$ and (b) the matrix

$$
G(\mathbf{x})=\left[\begin{array}{ll}
L_{g_{1}} L_{f}^{\sigma_{1}-1} h_{1} & L_{g_{2}} L_{f}^{\sigma_{1}-1} h_{1} \\
L_{g_{1}} L_{f}^{\sigma_{2}-1} h_{2} & L_{g_{2}} L_{f}^{\sigma_{2}-1} h_{2}
\end{array}\right]
$$

is nonsingular at $\mathbf{x}=\mathbf{x}_{0}$. 
By simple calculation, the input relative degree of system (6) is $(4,2)$ so that $G(\mathbf{x})$ can be rearranged as follows:

$$
G(\mathbf{x})=\left[\begin{array}{cc}
L_{g_{1}} L_{f}^{3} h_{1} & L_{g_{2}} L_{f}^{3} h \\
L_{g_{1}} L_{f} h_{2} & L_{g_{2}} L_{f} h_{2}
\end{array}\right] .
$$

Therefore, there exists a diffeomorphism

$$
\left[\begin{array}{c}
q \\
\dot{q} \\
\ddot{q} \\
\dddot{q} \\
\sigma \\
\dot{\sigma}
\end{array}\right]=\left[\begin{array}{c}
h_{1}(\mathbf{x}) \\
L_{f} h_{1}(\mathbf{x}) \\
L_{f}^{2} h_{1}(\mathbf{x}) \\
L_{f}^{3} h_{1}(\mathbf{x}) \\
h_{2}(\mathbf{x}) \\
L_{f} h_{2}(\mathbf{x})
\end{array}\right]
$$

that transforms system (6) into

$$
\left[\begin{array}{l}
q^{(4)} \\
\sigma^{(2)}
\end{array}\right]=F(\mathbf{x})+G(\mathbf{x}) \mathbf{u}
$$

with $F: R^{6} \mapsto R^{2}$ being a vector field.

Let $\mathbf{e}_{q}:=\left[e_{q}, \dot{e}_{q}, \ddot{e}_{q}, \dddot{e}_{q}\right]^{T}$ and $\mathbf{e}_{\sigma}:=\left[e_{\sigma}, \dot{e}_{\sigma}\right]^{T}$. Define

$$
\begin{aligned}
& e_{s 1}=\left(\frac{d}{d t}+\lambda_{1}\right)^{3} e_{q}=\left[\lambda_{1}^{3}, 3 \lambda_{1}^{2}, 3 \lambda_{1}, 1\right] \mathbf{e}_{q} \\
& e_{s 2}=\left(\frac{d}{d t}+\lambda_{2}\right) e_{\sigma}=\left[\lambda_{2}, 1\right] \mathbf{e}_{\sigma}
\end{aligned}
$$

with $\lambda_{1}, \lambda_{2} \in R^{+}$. Then, one gets

$$
\dot{\mathbf{e}}_{s}=F(\mathbf{x})+G(\mathbf{x}) \mathbf{u}+\mathbf{v}
$$

with $\mathbf{e}_{s}:=\left[e_{s 1}, e_{s 2}\right]^{T}, \mathbf{v}=\left[v_{1}, v_{2}\right]^{T}, v_{1}:=\left[0, \lambda_{1}^{3}, 3 \lambda_{1}^{2}, 3 \lambda_{1}\right] \mathbf{e}_{q}-$ $q_{d}^{(4)}$, and $v_{2}:=\left[0, \lambda_{2}\right] \mathbf{e}_{q}-\ddot{\sigma}_{d}$. A feedback linearization-based ideal control law is given by

$$
\mathbf{u}=G^{-1}(\mathbf{x})\left(-K \mathbf{e}_{s}-F(\mathbf{x})-\mathbf{v}\right)
$$

with $K \in R^{2 \times 2}$ being a positive-definite diagonal matrix of control gains.

3.3. Adaptive Neural Network Control. As $F(\mathbf{x})$ in control law (14) is not exactly known, a radial basis function- (RBF-) NN represented as follows:

$$
\widehat{f}(\mathbf{x}, \widehat{W})=\Phi^{T}(\mathbf{x}) \widehat{W}
$$

is applied to approximate $F(\mathbf{x})$, where $\widehat{W}=\left[\widehat{W}_{1}, \widehat{W}_{2}\right] \in R^{N \times 2}$ with $\widehat{W}_{i}=\left[\widehat{w}_{i 1}, \widehat{w}_{i 2}, \ldots, \widehat{w}_{i N}\right]^{T}(i=1,2)$ is a matrix of NN weights, $\Phi(\mathbf{x})=\left[\phi_{1}(\mathbf{x}), \phi_{2}(\mathbf{x}), \ldots, \phi_{N}(\mathbf{x})\right]^{T} \in R^{N}$ is a vector of regression functions, $N$ is the number of neural nodes, and $\phi_{j}(\mathbf{x}): R^{6} \mapsto R$ are commonly chosen to be Gaussian RBFs:

$$
\phi_{j}(\mathbf{x})=\exp \left(-\frac{\left\|\mathbf{x}-\mathbf{c}_{j}\right\|^{2}}{\left(2 r_{j}^{2}\right)}\right)
$$

with $j=1,2, \ldots, N$ and $\mathbf{c}_{j}=\left[c_{1 j}, c_{2 j}\right]^{T}$, where $c_{i j} \in R(i=$ 1,2) and $r_{j} \in R^{+}$are centers and widths of the Gaussian functions, respectively.

Let $\Omega_{w}:=\left\{\widehat{W} \mid\|\widehat{W}\|_{F} \leq c_{w}\right\}$ and $\Omega_{x}:=\left\{\mathbf{x} \mid\|\mathbf{x}\| \leq c_{x}\right\}$. Define an optimal NN approximation error

$$
\varepsilon(\mathbf{x}):=f(\mathbf{x})-\widehat{f}\left(\mathbf{x}, W^{*}\right),
$$

with a constant matrix of optimal weights

$$
W^{*}:=\underset{\widehat{W} \in \Omega_{c_{w}}}{\arg \min }\left\{\sup _{\mathbf{x} \in \Omega_{c_{x}}}|f(\mathbf{x})-\widehat{f}(\mathbf{x}, \widehat{W})|\right\} .
$$

According to the universal approximation property of RBFNNs, given any small constant $\varepsilon^{*} \in \mathbb{R}^{+}$, there is a sufficiently large $N$ so that $|\varepsilon(\mathbf{x})| \leq \varepsilon^{*}, \forall \mathbf{x} \in \Omega_{x}[41]$.

Assumption 4. There exists a matrix $G_{0}=\operatorname{diag}\left(g_{10}, g_{20}\right)$ with $g_{10}, g_{20} \in R^{+}$such that $G(\mathbf{x}) \geq G_{0}$ holds.

Now, the actual control law is designed as follows:

$$
\mathbf{u}=G_{0}^{-1}\left(-K \mathbf{e}_{s}-\Phi^{T}(\mathbf{x}) \widehat{W}-\mathbf{v}\right),
$$

and the update law of $\widehat{W}$ is given by

$$
\dot{\widehat{W}}=\Gamma \mathscr{P}\left(\Phi(\mathbf{x}) \mathbf{e}_{s}\right) \text {, }
$$

where $\Gamma \in R^{2 \times 2}$ is a positive-definite matrix of learning rates, and $\mathscr{P}$ is a projection operator given by

$$
\begin{aligned}
& \mathscr{P}(\bullet) \\
& = \begin{cases}\bullet, & \text { if }\|\widehat{W}\|_{F}<c_{w} \text { or }\|\widehat{W}\|_{F}=c_{w} \& \widehat{W}^{T} \bullet \leq 0 \\
\bullet-\frac{\widehat{W} \widehat{W}^{T}}{\|\widehat{W}\|_{F}^{2}} \bullet, & \text { otherwise. }\end{cases}
\end{aligned}
$$

Note that the choice of the gain matrix $G_{0}$ is based on a rough estimation of the inertia matrices $M, B_{1}$, and $B_{2}$, where the exact values of $M, B_{1}$, and $B_{2}$ are not required in practice. It follows from the standard NNAC result [41] that system (11) driven by control law (19) with (20) achieves practical asymptotic stability in the sense that $e_{q}$ and $e_{\sigma}$ converge to small neighborhoods of zero determined by $K$ and $\varepsilon^{*}$.

\section{Numerical Results}

A general specification of SVSAs in Table 1 [15] is used for simulation. The construction of the proposed NNAC follows the following steps: (1) to construct regression functions $\phi_{j}(\mathbf{x})$ in (16), select three Gaussian functions to cover each universe of $\Omega_{x}$ such that $j=1$ to 729 ; (2) set the filtered error parameters $\lambda_{1}=\lambda_{2}=20$ in (12); (3) set the lower bound $G_{0}=$ $\operatorname{diag}(50000,1000)$ and the control gain $K=\operatorname{diag}(20,20)$ for the control law in (19); (4) set the learning rate matrix $\Gamma=$ $\operatorname{diag}(300,100)$ for the adaptive law in $(20)$.

A tracking task with the desired position $q_{d}(t)=$ $120 \sin (\pi t)\left({ }^{\circ}\right)$ and the desired stiffness $\sigma_{d}(t)=50 \sin (1.25 \pi+$ 
TABLE 1: SVSA parameter specifications.

\begin{tabular}{lcc}
\hline Description & Symbol (unit) & Value \\
\hline $\begin{array}{l}\text { Output link inertia } \\
\text { Motor M1 + gearbox }+\end{array}$ & $M\left(\mathrm{~kg} \cdot \mathrm{m}^{2}\right)$ & 0.0063 \\
intermediate inertia & $B_{1}\left(\mathrm{~kg} \cdot \mathrm{m}^{2}\right)$ & 0.0134 \\
Motor M2 + gearbox + & $B_{2}\left(\mathrm{~kg} \cdot \mathrm{m}^{2}\right)$ & 0.0110 \\
ASRM inertia & $D(\mathrm{~N} \cdot \mathrm{ms} / \mathrm{rad})$ & $1.6 * 10^{-3}$ \\
Output link damping & $D_{1}(\mathrm{~N} \cdot \mathrm{ms} / \mathrm{rad})$ & $3.0 * 10^{-3}$ \\
Motor M1 damping & $D_{2}(\mathrm{~N} \cdot \mathrm{ms} / \mathrm{rad})$ & $2.4 * 10^{-3}$ \\
Motor M2 damping & $K s(\mathrm{~N} / \mathrm{m})$ & 3764.5 \\
Inherent spring stiffness & $R(\mathrm{~m})$ & 0.05 \\
Radius (mm) & $(\mathrm{deg} \cdot)$ & $0-360$ \\
Range of motion & $(\mathrm{deg} \cdot)$ & $0-30$ \\
Range of deflection angle & $(\mathrm{N} \cdot \mathrm{m} / \mathrm{rad})$ & $1.7-150.5$ \\
Range of stiffness & &
\end{tabular}
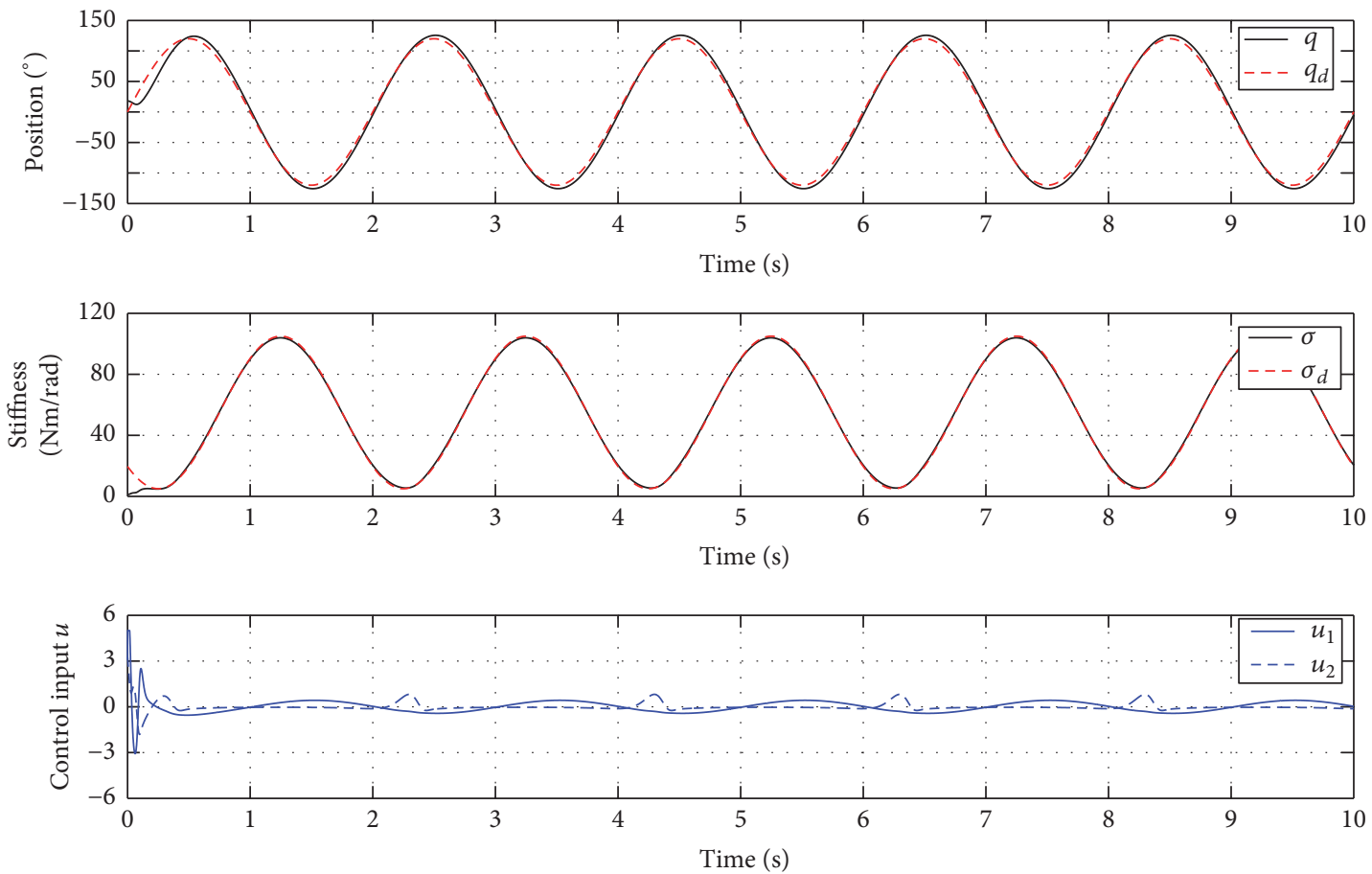

FIgURE 2: Control trajectories by the PD control without load.

$\pi t)+55(\mathrm{Nm} / \mathrm{rad}$ ) (frequency $0.5 \mathrm{~Hz}$ ) is chosen to show the tracking performance of the proposed controller for SVSAs, where the desired stiffness $\sigma_{d}$ ranges between 5 and $105 \mathrm{Nm} /$ rad. To make comparisons, a PD controller is selected as a baseline controller, where $\mathrm{PD}$ gains are optimized to minimize errors under a reasonable control $\mathbf{u}$.

Tracking results by the PD control and the proposed NNAC are demonstrated in Figures 2 and 3, respectively, and comparisons of system errors are given in Figure 5, where no load is applied on the actuator in this case. It is shown that the proposed controller achieves a much higher position tracking accuracy than the PD control under a comparable control input $\mathbf{u}$ despite the change of the stiffness. The position tracking accuracies by the PD control and the proposed NNAC are about $0.4547^{\circ}$ and $7.3490^{\circ}$, respectively. The stiffness tracking result also shows better performance of the proposed NNAC than the PD control, where the stiffness tracking accuracy reduces from $1.1200 \mathrm{Nm} / \mathrm{rad}$ to $0.2857 \mathrm{Nm} / \mathrm{rad}$. In addition, Figure 4 shows that the norms of the NN weights $\widehat{W}_{1}$ and $\widehat{W}_{2}$ converge to some constants, which implies that an exact estimation of the system uncertainty is achieved according to the NN learning theory [38].

To investigate the adaptability of the proposed NNAC, a $2 \mathrm{~kg}$ load is added to the link at $t=15 \mathrm{~s}$. System errors 

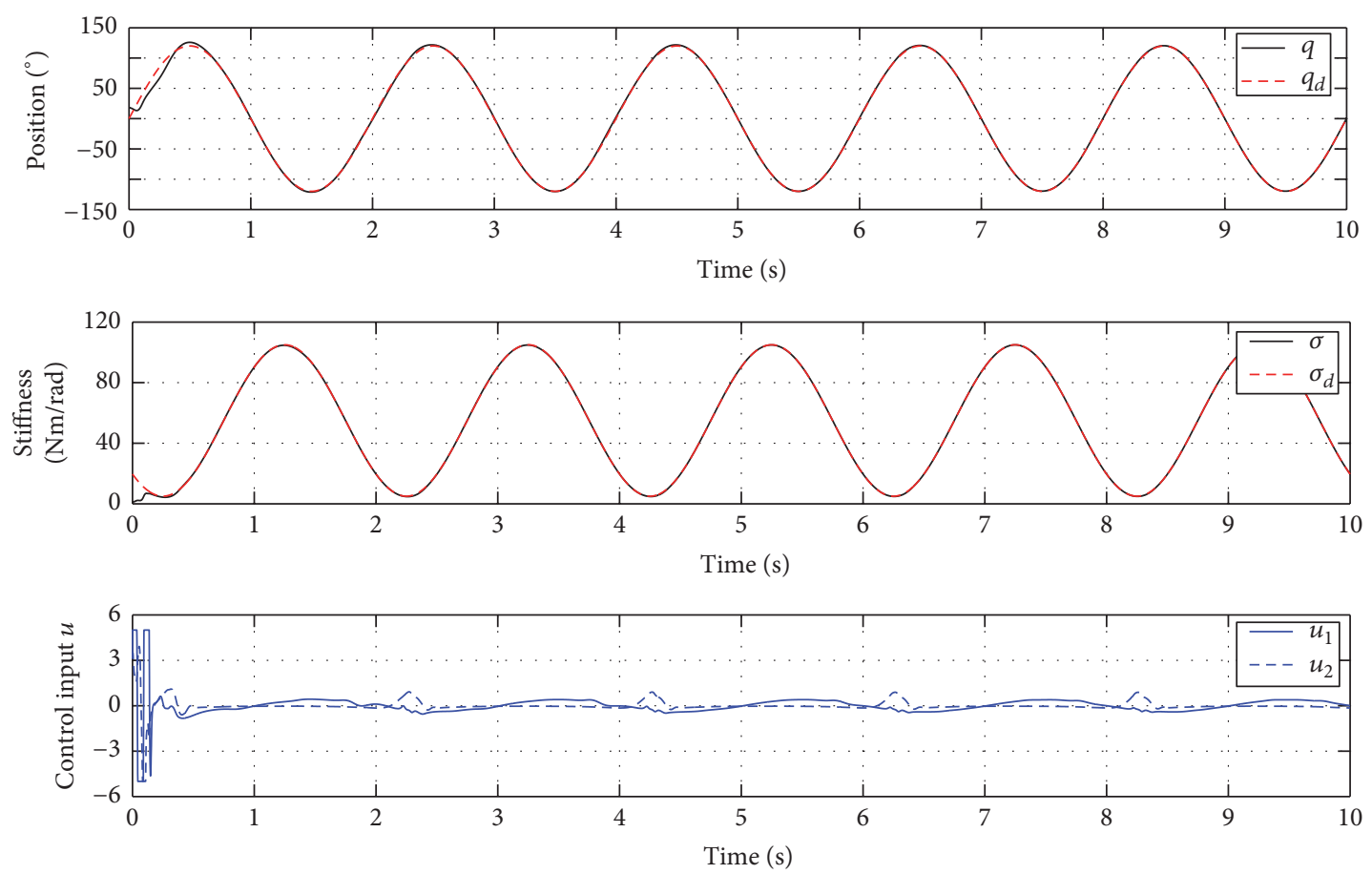

FIgURE 3: Control trajectories by the proposed NNAC without load.
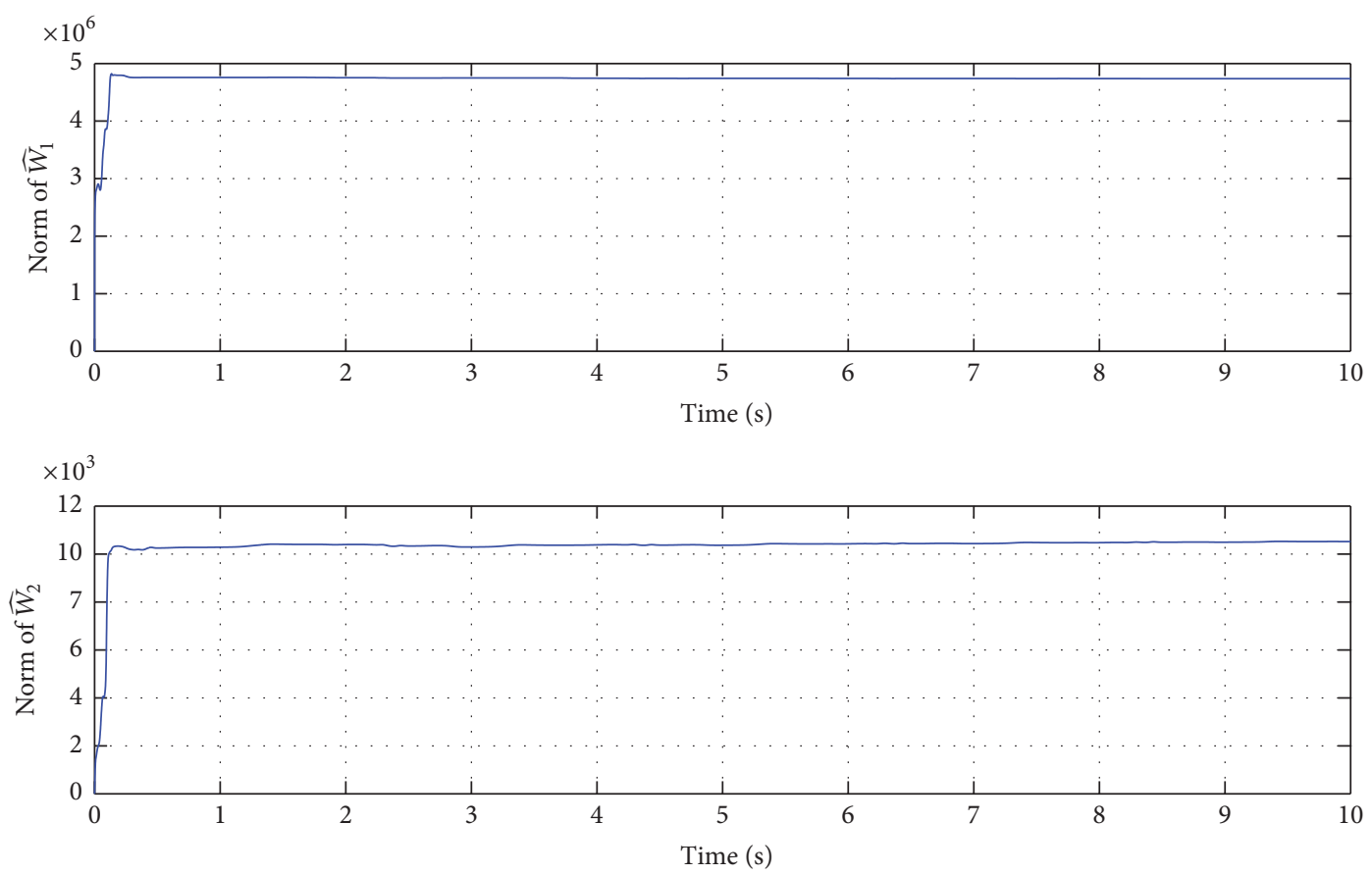

FIGURE 4: Evaluations of NN weights without load.

and control inputs within $30 \mathrm{~s}$ are depicted in Figure 6. It is shown that, at the time $15 \mathrm{~s}$, both the position error $e_{q}$ and the stiffness error $e_{\sigma}$ increase dramatically but then reduce gradually to small values. After an adaptation about $15 \mathrm{~s}$, the high position tracking accuracy is still maintained by the proposed NNAC, where the maximum position error $e_{q}\left(0.8744^{\circ}\right)$ is even smaller than that before adding the load $\left(0.9265^{\circ}\right)$. The adaptability of the proposed NNAC is apparent as position error $e_{q}$ continues decreasing to small range despite the unknown load added. Note that adding loads does not affect the stiffness tracking accuracy.

\section{Conclusions}

This paper has successfully applied a NNAC method to control SVSAs. Simulation results verify the ability of the 

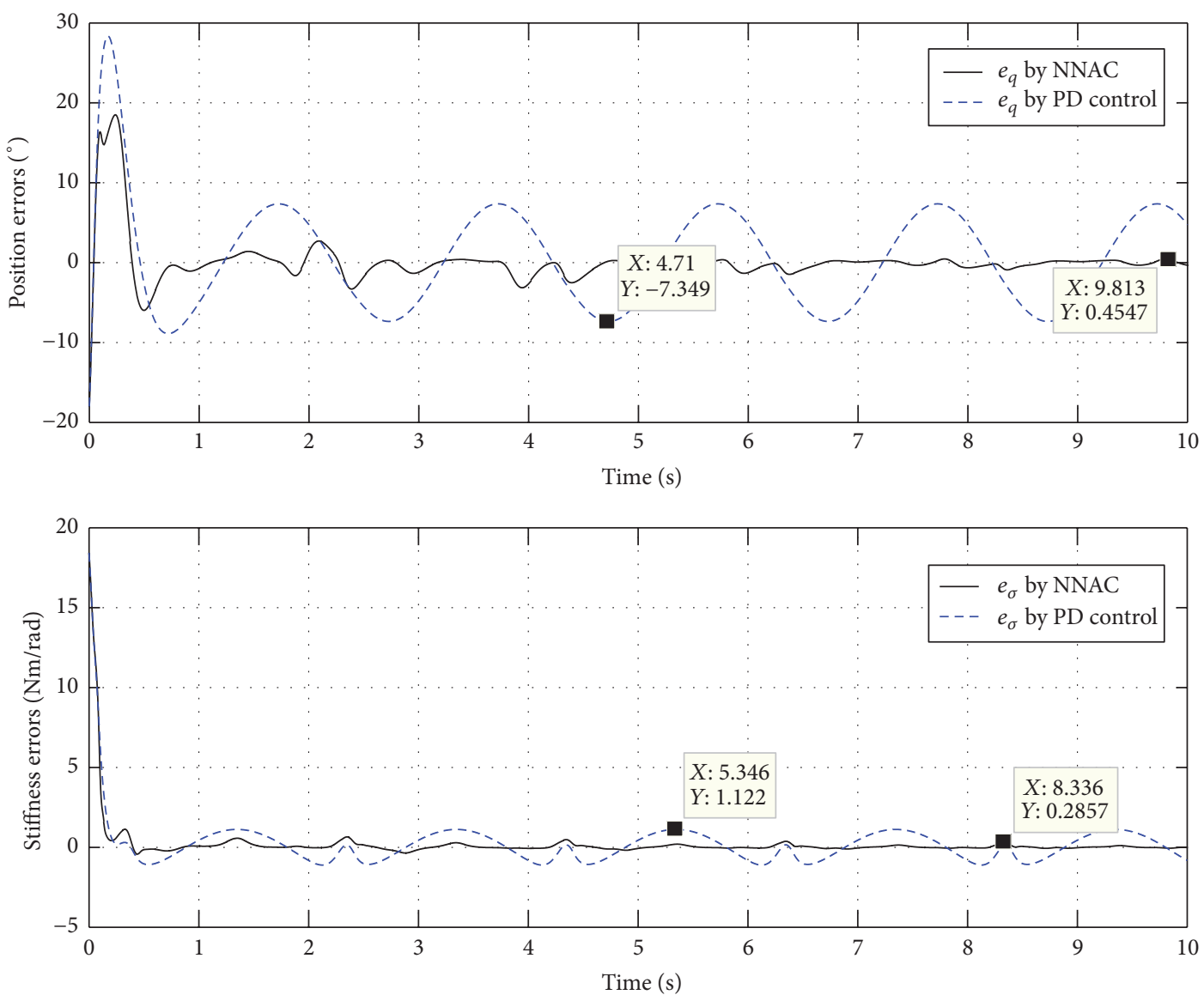

FIgURE 5: Comparisons of system errors without load.
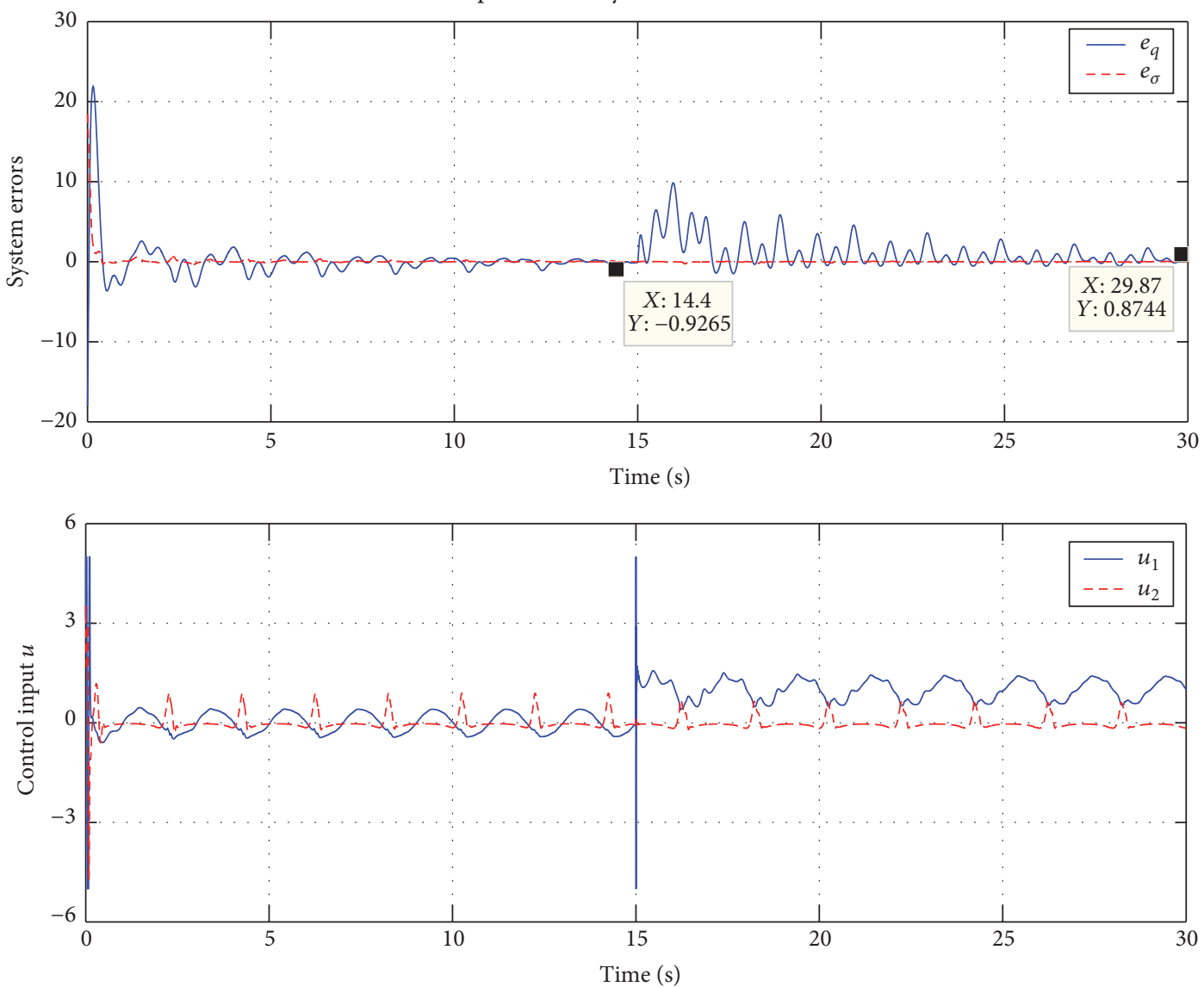

FIGURE 6: Control trajectories by the proposed NNAC with load. 
proposed approach to cope with system variability, by showing remarkable control performances for both position and stiffness tracking under load changes. In future work, the practicability of the proposed NNAC would be enhanced by the composite learning [42] and the observer design [43], and experimental validity of the proposed controller will be carried out on a physical SVSA system.

\section{Conflicts of Interest}

The authors declare that they have no conflicts of interest.

\section{Acknowledgments}

This work was supported by the National Natural Science Foundation of China (nos. 61703295, 51605339, and 51675385), the Natural Science Foundation of Jiangsu Province (no. BK20150385), the Natural Science Foundation of Hubei Province (no. 2017CFB496), the China Postdoctoral Science Foundation (nos. 2016M592382 and 2017T100573), Wuhan Youth Science and Technology Dawn Plan (no. 2017050304010304), and the Fundamental Research Funds for the Central Universities (no. 2042016kf0021).

\section{References}

[1] B. Vanderborght, A. Albu-Schaeffer, A. Bicchi et al., "Variable impedance actuators: a review," Robotics and Autonomous Systems, vol. 61, no. 12, pp. 1601-1614, 2013.

[2] S. Wolf and G. Hirzinger, "A new variable stiffness design: Matching requirements of the next robot generation," in Proceedings of the 2008 IEEE International Conference on Robotics and Automation, ICRA 2008, pp. 1741-1746, usa, May 2008.

[3] S. Wolf, G. Grioli, O. Eiberger et al., "Variable Stiffness Actuators: Review on Design and Components," IEEE/ASME Transactions on Mechatronics, vol. 21, no. 5, pp. 2418-2430, 2016.

[4] H. Yu, S. Huang, G. Chen, and N. Thakor, "Control design of a novel compliant actuator for rehabilitation robots," Mechatronics, vol. 23, no. 8, pp. 1072-1083, 2013.

[5] Z. Guo, H. Yu, and L.-B. Wee, "Design of a novel compliant differential Shape Memory Alloy actuator," in Proceedings of the 2013 26th IEEE/RSJ International Conference on Intelligent Robots and Systems: New Horizon, IROS 2013, pp. 4925-4930, jpn, November 2013.

[6] Z. Guo, Y. Pan, L. B. Wee, and H. Yu, "Design and control of a novel compliant differential shape memory alloy actuator," Sensors and Actuators A: Physical, vol. 225, pp. 71-80, 2015.

[7] Y. Pan, Z. Guo, X. Li, and H. Yu, "Output-feedback adaptive neural control of a compliant differential SMA actuator," IEEE Transactions on Control Systems Technology, vol. 25, no. 6, pp. 2202-2210, 2017.

[8] G. Grioli, S. Wolf, M. Garabini et al., "Variable stiffness actuators: the user's point of view," International Journal of Robotics Research, vol. 34, no. 6, pp. 727-743, 2015.

[9] H. Q. Vu, X. Yu, F. Iida, and R. Pfeifer, "Improving Energy Efficiency of Hopping Locomotion by Using a Variable Stiffness Actuator," IEEE/ASME Transactions on Mechatronics, vol. 21, no. 1, pp. 472-486, 2016.

[10] R. Schiavi, G. Grioli, S. Sen, and A. Bicchi, "VSA-II: A novel prototype of variable stiffness actuator for safe and performing robots interacting with humans," in Proceedings of the 2008 IEEE International Conference on Robotics and Automation, ICRA 2008, pp. 2171-2176, usa, May 2008.

[11] L. C. Visser, R. Carloni, and S. Stramigioli, "Energy-efficient variable stiffness actuators," IEEE Transactions on Robotics, vol. 27, no. 5, pp. 865-875, 2011.

[12] A. Jafari, N. G. Tsagarakis, B. Vanderborght, and D. G. Caldwell, "A novel actuator with adjustable stiffness (AwAS)," in Proceedings of the 23rd IEEE/RSJ 2010 International Conference on Intelligent Robots and Systems, IROS 2010, pp. 4201-4206, twn, October 2010.

[13] A. Jafari, N. G. Tsagarakis, I. Sardellitti, and D. G. Caldwell, "A new actuator with adjustable stiffness based on a variable ratio lever mechanism," IEEE/ASME Transactions on Mechatronics, vol. 19, no. 1, pp. 55-63, 2014.

[14] N. G. Tsagarakis, I. Sardellitti, and D. G. Caldwell, "A new variable stiffness actuator (CompAct-VSA): design and modelling," in Proceedings of the 2011 IEEE/RSJ International Conference on Intelligent Robots and Systems: Celebrating 50 Years of Robotics (IROS '11), pp. 378-383, IEEE, San Francisco, Calif, USA, September 2011.

[15] J. Sun, Y. Zhang, C. Zhang, Z. Guo, and X. Xiao, "Mechanical design of a compact Serial Variable Stiffness Actuator (SVSA) based on lever mechanism," in Proceedings of the 2017 IEEE International Conference on Robotics and Automation (ICRA), pp. 33-38, Singapore, May 2017.

[16] G. Tonietti, R. Schiavi, and A. Bicchi, "Design and control of a variable stiffness actuator for safe and fast physical human/robot interaction," in Proceedings of the 2005 IEEE International Conference on Robotics and Automation, pp. 526531, esp, April 2005.

[17] G. Palli, C. Melchiorri, and A. De Luca, "On the feedback linearization of robots with variable joint stiffness," in Proceedings of the 2008 IEEE International Conference on Robotics and Automation, ICRA 2008, pp. 1753-1759, usa, May 2008.

[18] G. Buondonno and A. De Luca, "Efficient Computation of Inverse Dynamics and Feedback Linearization for VSA-Based Robots," IEEE Robotics and Automation Letters, vol. 1, no. 2, pp. 908-915, 2016.

[19] I. Sardellitti, G. A. Medrano-Cerda, N. Tsagarakis, A. Jafari, and D. G. Caldwell, "Gain scheduling control for a class of variable stiffness actuators based on lever mechanisms," IEEE Transactions on Robotics, vol. 29, no. 3, pp. 791-798, 2013.

[20] F. Petit and A. Albu-Schäffer, "State feedback damping control for a multi DOF variable stiffness robot arm," in Proceedings of the 2011 IEEE International Conference on Robotics and Automation, ICRA 2011, pp. 5561-5567, chn, May 2011.

[21] F. Petit, A. Daasch, and A. Albu-Schaffer, "Backstepping Control of Variable Stiffness Robots," IEEE Transactions on Control Systems Technology, vol. 23, no. 6, pp. 2195-2202, 2015.

[22] A. Zhakatayev, M. Rubagotti, and H. A. Varol, "Closed-Loop Control of Variable Stiffness Actuated Robots via Nonlinear Model Predictive Control," IEEE Access, vol. 3, pp. 235-248, 2015.

[23] G. Palli and C. Melchiorri, "Output-based control of robots with variable stiffness actuation," Journal of Robotics, vol. 2011, Article ID 735407, 15 pages, 2011.

[24] S. Ulrich, J. Z. Sasiadek, and I. Barkana, "Nonlinear adaptive output feedback control of flexible-joint space manipulators with joint stiffness uncertainties," Journal of Guidance, Control, and Dynamics, vol. 37, no. 6, pp. 1961-1975, 2014. 
[25] T. Dierks, B. Brenner, and S. Jagannathan, "Neural networkbased optimal control of mobile robot formations with reduced information exchange," IEEE Transactions on Control Systems Technology, vol. 21, no. 4, pp. 1407-1415, 2013.

[26] Y. Li, S. S. Ge, Q. Zhang, and T. . Lee, "Neural networks impedance control of robots interacting with environments," IET Control Theory \& Applications, vol. 7, no. 11, pp. 1509-1519, 2013.

[27] X. Li and C. C. Cheah, "Adaptive neural network control of robot based on a unified objective bound," IEEE Transactions on Control Systems Technology, vol. 22, no. 3, pp. 1032-1043, 2014.

[28] M. Hamdy and G. EL-Ghazaly, "Adaptive neural decentralized control for strict feedback nonlinear interconnected systems via backstepping," Neural Computing and Applications, vol. 24, no. 2, pp. 259-269, 2014.

[29] R. Mei and C. Yu, "Adaptive Neural Output Feedback Control for Uncertain Robot Manipulators with Input Saturation," Complexity, Article ID 7413642, 12 pages, 2017.

[30] B. Xu and P. Zhang, "Minimal-learning-parameter technique based adaptive neural sliding mode control of MEMS gyroscope," Complexity, vol. 2017, Article ID 6019175, 8 pages, 2017.

[31] Y. Pan, Y. Liu, B. Xu, and H. Yu, "Hybrid feedback feedforward: An efficient design of adaptive neural network control," Neural Networks, vol. 76, pp. 122-134, 2016.

[32] C. Yang, X. Wang, Z. Li, Y. Li, and C. Su, "Teleoperation control based on combination of wave variable and neural networks," IEEE Transactions on Systems, Man, and Cybernetics: Systems, vol. 47, no. 8, pp. 2125-2136, 2017.

[33] M. Hamdy, S. Abd-Elhaleem, and M. A. Fkirin, "Time-varying delay compensation for a class of nonlinear control systems over network via $\mathrm{H} \infty$ adaptive fuzzy controller," IEEE Transactions on Systems, Man, and Cybernetics: Systems, vol. 47, no. 8, pp. 2114-2124, 2017.

[34] M. Boukens, A. Boukabou, and M. Chadli, "Robust adaptive neural network-based trajectory tracking control approach for nonholonomic electrically driven mobile robots," Robotics and Autonomous Systems, vol. 92, pp. 30-40, 2017.

[35] G. W. Woodford, M. C. du Plessis, and C. J. Pretorius, "Concurrent controller and Simulator Neural Network development for a snake-like robot in Evolutionary Robotics," Robotics and Autonomous Systems, vol. 88, pp. 37-50, 2017.

[36] C. Yang, X. Wang, L. Cheng, and H. Ma, "Neural-learningbased telerobot control with guaranteed performance," IEEE Transactions on Cybernetics, vol. 47, no. 10, pp. 3148-3159, 2017.

[37] C. Yang, K. Huang, H. Cheng, Y. Li, and C. Su, "Haptic identification by ELM-controlled uncertain manipulator," IEEE Transactions on Systems, Man, and Cybernetics: Systems, vol. 47, no. 8, pp. 2398-2409, 2017.

[38] Y. Pan and H. Yu, "Biomimetic hybrid feedback feedforward neural-network learning control," IEEE Transactions on Neural Networks and Learning Systems, vol. 28, no. 6, pp. 1481-1487, 2017.

[39] S. Huh, G. Tonietti, and A. Bicchi, "Neural network based robust adaptive control for a variable stiffness actuator," in Proceedings of the 2008 Mediterranean Conference on Control and Automation, MED'08, pp. 1028-1034, Ajaccio, France, June 2008.

[40] H. K. Khalil, Nonlinear Systems, Prentice-Hall, Englewood Cliffs, NJ, USA, 2nd edition, 2002.

[41] J. A. Farrell and M. M. Polycarpou, Adaptive Approximation Based Control: Unifying Neural, Fuzzy and Traditional Adaptive Approximation Approaches, Wiley, Hoboken, NJ, USA, 2006.
[42] Y. Pan and H. Yu, "Composite learning from adaptive dynamic surface control," IEEE Transactions on Automatic Control, vol. 61, no. 9, pp. 2603-2609, 2016.

[43] Y. Pan, T. Sun, and H. Yu, "Peaking-free output-feedback adaptive neural control under a nonseparation principle," IEEE Transactions on Neural Networks and Learning Systems, vol. 26, no. 12, pp. 3097-3108, 2015. 


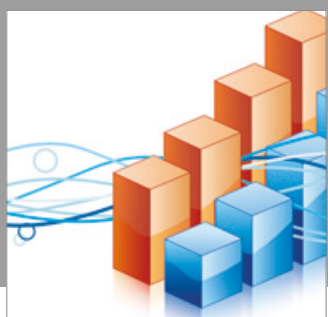

Advances in

Operations Research

vatersals

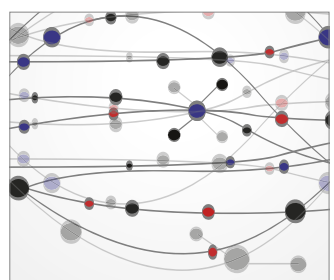

\section{The Scientific} World Journal
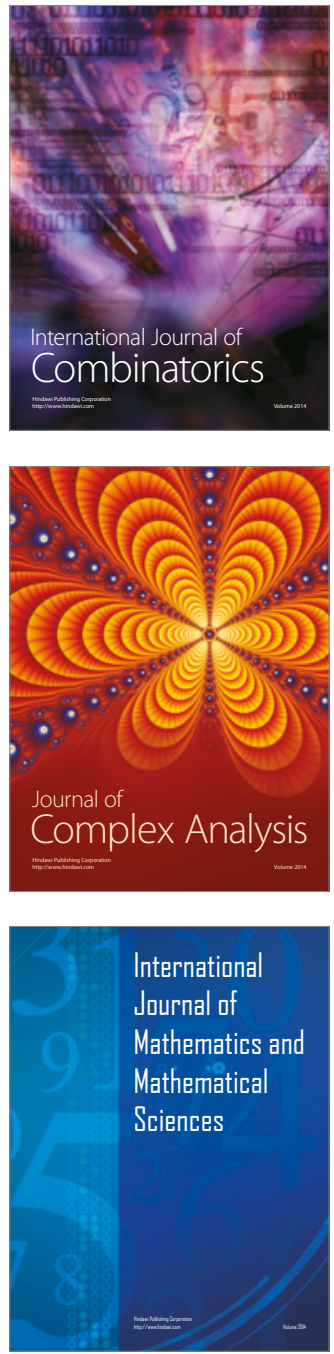
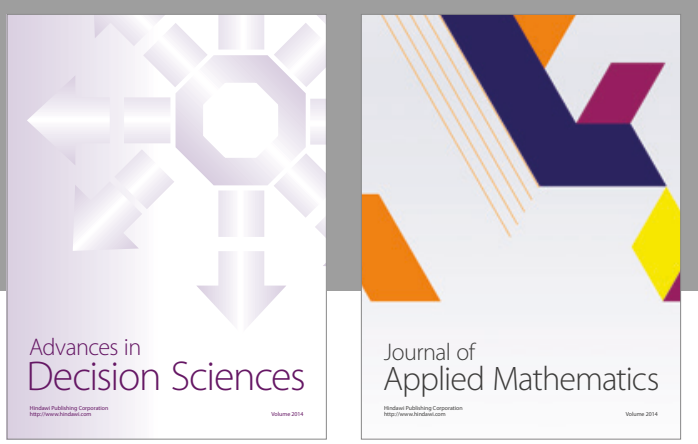

Algebra

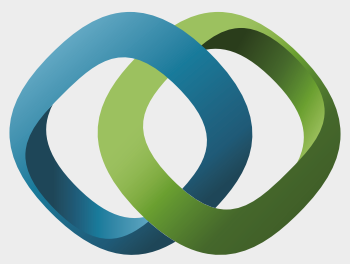

\section{Hindawi}

Submit your manuscripts at

https://www.hindawi.com
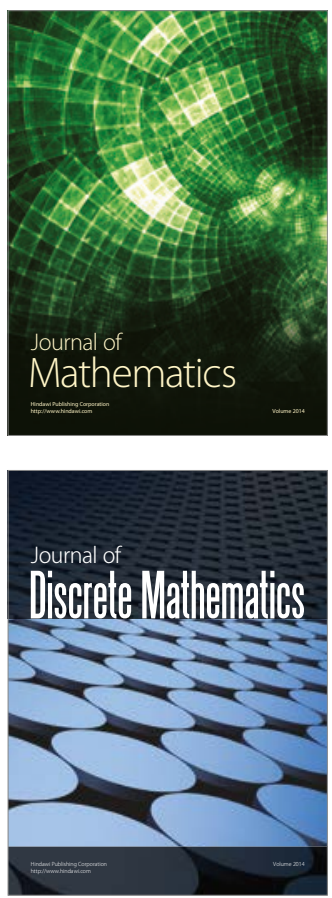

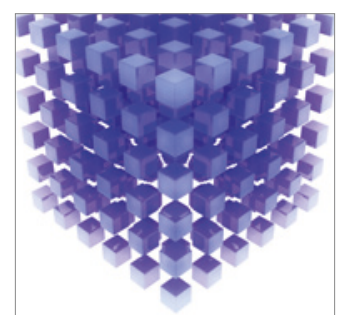

Mathematical Problems in Engineering
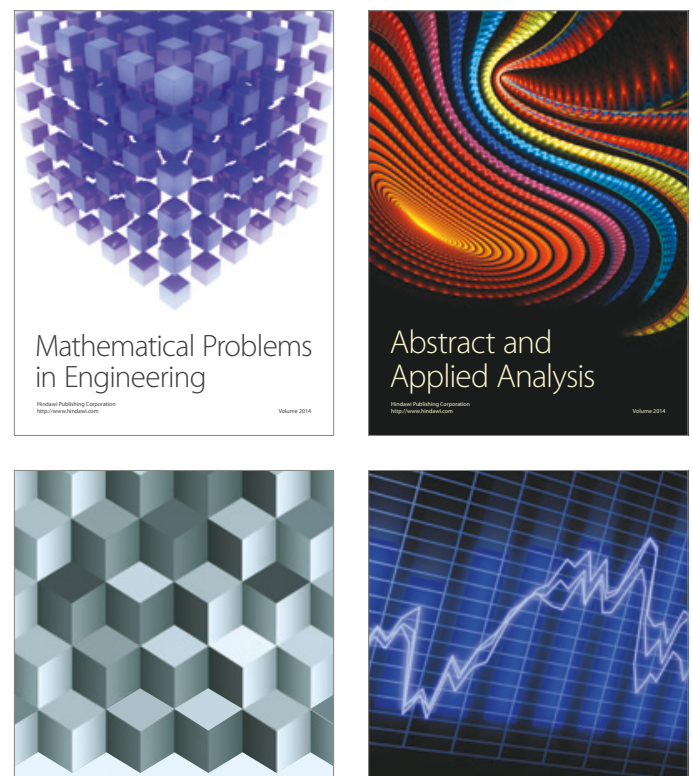

Journal of

Function Spaces

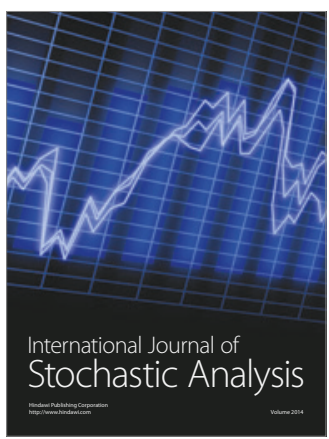

Probability and Statistics
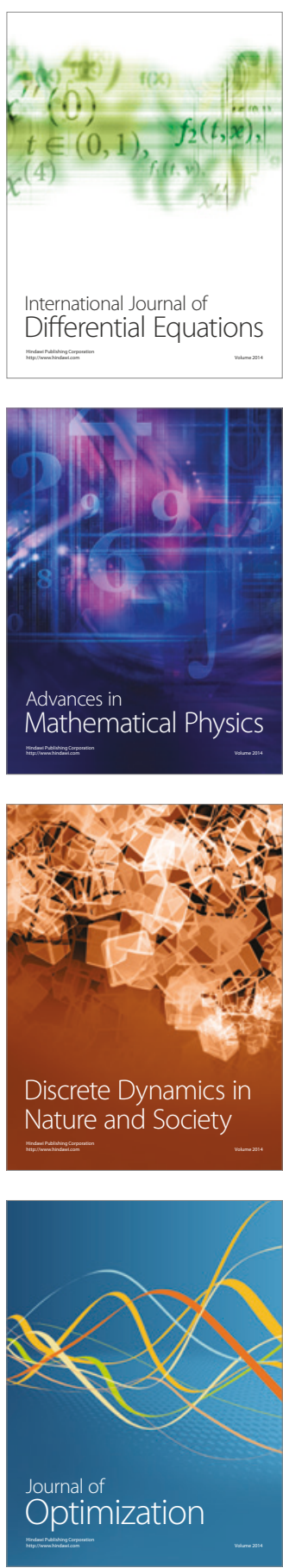